\title{
¿Qué se dice cuando se dice cultura? Notas sobre el nombre de un problema
}

\author{
Pedro Güell ${ }^{1}$
}

\begin{abstract}
Resumen
A pesar de su importancia, el concepto de cultura ha tenido una trayectoria dificil en las Ciencias Sociales. Sus usos son múltiples y sus definiciones ambiguas. La hipótesis general del presente artículo es que el término cultura no tiene la pretensión de ser la referencia a un objeto, sino que busca establecer una perspectiva o un marco amplio para definir y enfrentar a la sociedad moderna como una paradoja: la escisión entre subjetividad y orden social. Se señala, por una parte, que esa paradoja tiene su origen en la crisis de la promesa de la Ilustración sobre la autonomía del sujeto y que el concepto de cultura fue una de las vías privilegiadas para definir y enfrentar esa crisis. Por la otra, se afirma que en la sociología el uso del concepto de cultura remite a la identificación de una tensión entre el fundamento subjetivo del sentido del mundo y la autonomía objetiva de las dinámicas que producen el orden y la integración social, siendo a la vez un conjunto de propuestas de mediación entre ambas. Con este fin se analiza el uso y significado del concepto en la obra de Simmel, Weber y Durkheim. Finalmente, se argumenta sobre las razones que permiten suponer que el uso y significado del concepto de cultura en esos autores puede encontrarse también en varios de sus sucesores.
\end{abstract}

\section{What do we mean when we talk about culture? Notes about the name of a problem}

Abstract

Besides its importance, the concept of culture has had a difficult path through the Social Sciences. Its uses are multiple and its definitions ambiguous. This paper's general hypothesis is that the concept of culture does not pretend to be the reference of an object, but seeks to establish a perspective or a wide frame to define and face modern society as a paradox: the division between subjectivity and social order. On the one hand, this article indicates that this paradox has its origin in the crisis of the Enlightenment's promise regarding subject's autonomy and that the concept of culture was one of the privileged routes to define and face that crisis. On the other hand, it affirms that the use of the concept of culture in sociology refers to the identification of a tension between the subjective foundations of the world's meaning and the objective autonomy of the dynamics that produce order and social integration, being, at the same time, a group of mediation proposals between them. With this purpose, this paper analyzes the use and meaning of the concept present in Simmel's, Weber's and Durkheim's works. Finally, it presents the reasons that allow supposing that the use and meaning of the concept of culture in those authors can also be found in many of their successors.

${ }^{1}$ Doctor en Sociología de la Universidad de Erlangen-Nürnberg, Alemania. Decano de la Facultad de Ciencias Sociales de la Universidad Alberto Hurtado e Investigador del Equipo de Desarrollo Humano del Programa de Naciones Unidas para el Desarrollo (pguell@uahurtado.cl). 


\section{La indefinición del concepto de cultura}

La cultura ha dado muchos dolores de cabeza, comenzando por la palabra misma. De ella decía Raymond Williams, quien hizo de las ciencias de la cultura un arte: "Cultura. No sé cuantas veces he deseado no haber oído nunca la maldita palabra" (Williams 1979: 174). Luhmann, por su parte, remató afirmando que cultura era uno de los peores conceptos que alguna vez se hubieran creado (Luhmann 1995).

Muchos de los que han estado de acuerdo con esta queja, señalan que la palabra aquella conduce a puras confusiones, entre otras cosas porque cada uno la define como quiere y nadie puede aportar un hecho empírico irrefutable que diga de una buena vez y sin ambigüedades lo que es y lo que no es cultura. En 1963 Kroeber y Cluckhohn hicieron un estudio para poner atajo a estos malestares. Reunieron en un corral varios cientos de conceptos de cultura que rondaban por el ambiente, les hicieron la autopsia para ver si llegaban a un ADN común y luego de mucho trabajo llegaron a la conclusión que, efectivamente, se trataba de un dolor de cabeza. En efecto, más allá de reducir un poco el desorden, la disección mostraba que se trataba de conceptos incompatibles. Como buenos positivistas que eran, le echaron la culpa no a la inexistencia del objeto cultura, sino a la debilidad del gremio para seguir las reglas que indican cómo se hace una buena definición conceptual acompañada de su correspondiente operacionalización empírica. En el fondo, la confusión e imprecisión era sólo un mal pasajero, se conocía la cura, ahora había que ponerse a trabajar o a esperar (Kroeber y Cluckhohn 1963). No resignados con el resultado, otros autores emprendieron similar trabajo arqueológico, aunque los resultado no fueron más alentadores (Bauman 2002, Reckwitz 2006).

Con el paso del tiempo las cosas sólo empeoraron. Si definirla ya era difícil en el marco del positivismo, que solo veía la cultura como un objeto específico entre otros, con la llegada del llamado 'giro cultural' de los años ochenta reinó el caos, pues todos los fenómenos sociales fueron definidos como textos significativos y, por lo tanto, como culturales. Desde esa perspectiva, cada día se descubren más cosas que son culturales, como los consumos, los cuerpos, los géneros, las industrias y las políticas relativas a los llamados 'bienes simbólicos' (Alexander 2000, Jameson 1999, Reckwitz 2004). Esta es la situación actual: si todo es cultura, cualquier cosa puede ser llamada cultural.

También hay autores que no se hacen dolores de cabeza con la palabra, porque la toman como un concepto histórico y no teórico o académico. Algo así como: 'no sé si tiene sentido o no, no es mi problema; pero alguna gente en algún período la ha usado como si tuviera sentido, ese es el objeto que hay que investigar, su uso, porque eso es un hecho empírico claro y distinto'. Pero acto seguido, se hacen problemas con los usos que se hace de ella. Unos dicen que sirvió de música para arrear un pueblo al fascismo, o para izar las banderas de la nación contra sus vecinos. Otros dirán 
que crea las ilusiones del consenso y esconde los conflictos, que sirve para alienar del dolor de las miserias, o que niega los cambios de la historia real. Más recientemente, unos declaran que sirve para crear un choque artificial entre civilizaciones, y otros que sirve para deslegitimar los roles naturales del hombre y de la mujer, es decir, para quitarle el piso a la ley natural y al buen orden.

En fin, unos expresan que el uso de la palabra cultura es malo porque crea la ilusión de las esencias inmutables, de un reino del espíritu no contaminado con las bajezas de este mundo, y otros señalan que es malo porque produce precisamente lo contrario: hace aparecer al orden y a las esencias de la vida como si fueran simples creaciones arbitrarias, reduciendo a la humanidad a la materialidad de la vida.Y, claro está, todos los que dicen estas cosas se crean sus propios conceptos de cultura para demostrar que la cultura no es lo que los otros dicen que es.

Pero la cultura no muere, por lo menos no la palabra ni los análisis culturales. Los seguidores del positivismo han continuado usando las mismas definiciones de cultura, aunque sus operacionalizaciones sean contradictorias, práctica en la cual los antropólogos llevan la delantera. Los hijos del 'giro cultural', por su parte, siguen hablando de aquello que es todo y nada específico al mismo tiempo. Luhman y sus seguidores aseguran que nunca ha existido algo así como cultura. Mientras tanto, nuevos teóricos como Margaret Archer (1988) o Andreas Reckwitz (2006) buscan caminos para superar los extravíos en torno al concepto de cultura y proponen definiciones hospitalarias capaces de acoger las diferencias y reducir los conflictos.

Como diría Galileo, a pesar de todo, se mueve. A pesar de la conciencia de la debilidad analítica, de la imprecisión empírica y de la ambivalencia política del concepto cultura, sigue siendo usado con deleite y pocos, realmente muy pocos, dudan de que exista algo así como la o las culturas y que se le hace un favor al esclarecimiento de la sociedad si se investigan sus dinámicas.

Y si esto es así, ¿qué necesidad se satisface cuando se habla de cultura, a nombre de la cultura o en perspectiva cultural? Tanta insistencia a pesar de las dificultades ha de tener algún motivo profundo. ¿Qué se dice cuando se dice cultura? A estas alturas debería estar relativamente claro que con esa palabra no parece posible identificar, sin fuertes imprecisiones, tautologías y supuestos metafisicos, un objeto con ese nombre dentro del catálogo de los hechos de la sociedad descrita como fenómeno real, ya sea real en el sentido de la naturaleza fisica de los positivistas, mental al modo de los hijos de la fenomenología, o biológica o cibernética al modo de los sistémicos. Tampoco, como se ha visto, parece que el problema pueda resolverse mejorando la lógica o la pertinencia empírica de las definiciones.

Frente a tanta confusión, por supuesto que estas notas no pretenden, ni podrían aunque lo quisieran, resolver el problema. Simplemente aspiran a sugerir algunas hipó- 
tesis en el campo de la historia de la teoría que permitan ofrecer una ampliación del campo de discusión. La hipótesis general del texto es que en su uso y estructura cultura no tiene la pretensión de ser la referencia a un objeto, sino que busca establecer una perspectiva o un marco amplio para definir y enfrentar a la sociedad moderna como una paradoja a la vez inevitable e ineludible: la escisión entre subjetividad y orden social. La cultura no da dolores de cabeza, su objeto es la modernidad como dolor de cabeza. Esto puede desagregarse en dos hipótesis parciales.

La primera hipótesis señala que la modernidad, como escisión problemática entre subjetividad y orden social, tiene su origen en la crisis de la promesa que la Ilustración le hizo al sujeto sobre su autonomía. Aquella se puede describir como la afirmación de que es posible una identidad entre la subjetividad, a la que se le asigna una capacidad para fundar desde sí misma la racionalidad del mundo, y la propia racionalidad inmanente que se le atribuye a la naturaleza, incluida a la sociedad dentro de ella. En la reflexión sobre la modernidad, la introducción del antiguo concepto de cultura sirvió precisamente para dar cuenta de las consecuencias de la crisis de esta promesa.

La segunda hipótesis indica que en la sociología la pretensión primaria del concepto de cultura no es identificar un objeto o dinámica dentro de la imagen predominantemente naturalizada de la sociedad que comienza a surgir desde mediados del siglo XIX. ¿Qué es lo que define el sentido de este concepto si se analiza su estructura y su uso? Se trata, por una parte, de la identificación de una tensión entre el fundamento subjetivo del sentido del mundo y la autonomía objetiva de las dinámicas que producen el orden y la integración social. Cultura sirve para identificar la paradoja moderna no como problema filosófico, sino como desafio real de las sociedades por lograr una mediación entre subjetividad y orden. Por otra parte, cultura es el espacio intelectual en que se formulan las teorías de esta mediación, sin la cual resulta difícil incluso pensar a la sociedad. Dentro de la historia de los empeños por encontrar gracias al concepto de cultura la ansiada mediación, se pueden reconocer recurrentes fracasos. Por lo mismo, al desarrollar sus teorías sobre la cultura, los autores suelen oscilar entre el énfasis en el sujeto y el énfasis en el orden, buscando así cerrar un círculo que se resiste a ello. Así, la historia de las teorías de la cultura es tan paradojal como su objeto.

En lo que sigue se tratará, de manera muy general, de mostrar la plausibilidad de estas hipótesis. En el caso de la primera, mediante una reconstrucción de la recepción moderna del concepto de cultura en el contexto de la reflexión filosófica sobre la crisis de la Ilustración. En el segundo caso, mediante un análisis del período inicial de la carrera del concepto o perspectiva cultural en las Ciencias Sociales a propósito de la obra de Simmel, Weber y Durkheim. Adicionalmente de manera muy sumaria se argumenta sobre las razones que permiten suponer que la estructura original del 
uso del concepto de cultura en esos autores puede encontrarse también en varios de sus sucesores.

\section{Recepción y gestación del concepto de cultura en la crisis de la Ilustración}

Cuando en 1911 Georg Simmel escribió en "El concepto y tragedia de la cultura" que el sujeto se ve "experimentando innumerables tragedias en esta profunda oposición de forma: entre la vida subjetiva que es incesante, pero temporalmente finita, $y$ sus contenidos, que una vez creados, son inamovibles pero válidos al margen del tiempo" y cuando luego concluyó "En medio de este dualismo habita la idea de cultura" (Simmel 2002 [1911]: 218), estaba resumiendo de una manera particular casi doscientos años de debate en torno a la sociedad moderna y poniéndolo como punto de partida de su sociología de la cultura.

El debate sobre la sociedad moderna no se inició con el deseo de deshacerse de la iglesia medieval y sus dioses, sino después, en el momento en que hubo que responder a la pregunta ¿y ahora sobre qué vamos a fundar la certeza de nuestro saber y la corrección de nuestro actuar? Descartes, uno de los padres de la promesa ilustrada, propuso hacia 1640 que el fundamento de la nueva realidad liberada del peso de los dioses y de las costumbres era la conciencia del sujeto individual. El individuo, en un acto de pura reflexión, podría identificar de manera 'clara y distinta' los principios de una razón que, al modo de una geometría, podían dar certeza al conocimiento y racionalidad al ordenamiento del mundo. Con él partió la promesa que definió a la Ilustración. La subjetividad del individuo es el rey, y ello gracias a que es soberana sobre los hechos de la naturaleza. El corazón de esa promesa descansaba en el supuesto de la existencia de una identidad entre la razón cuyos principios descubría el intelecto y la razón que subyacía al orden de la naturaleza.

No pasó mucho tiempo antes de que otros pensadores descubrieran que esa promesa era un problema. Sin contar a los nostálgicos del pasado, entre los propios hijos de la Ilustración, como Hume, Kant o Hegel, muy pronto se vio que resultaba muy dificil de justificar una razón que sirviera de puente entre el modo de funcionamiento de la subjetividad y el modo de existencia de los hechos de la naturaleza. En un acto de humildad, los filósofos de la autonomía subjetiva fueron reconociendo que la naturaleza tenía sus propias reglas que no eran conocibles por sí mismas. Para relacionarse con ella sólo quedaba apostar al juego de las interpretaciones, siempre inciertas, o esperar que el espíritu de la historia juntara algún día razón y naturaleza. Tomaron con ello distancia de la naturaleza, pero no renunciaron a la afirmación radical de la autonomía del sujeto como fundamento de la razón (Gellner 2005, Bürger 2001, Taubes 2007). 
Mientras los filósofos se agobiaban con estos problemas, la nueva profesión de los científicos y técnicos decidió resolver las dudas haciendo pruebas y aprendiendo de los errores. En vez de preguntarse si la razón geométrica tenía un buen fundamento en el sujeto, se preguntaron si el resultado de aplicarla a la naturaleza era útil. Si lo era, es decir si permitía el dominio, entonces tenía fundamento, aunque no pudieran justificarla subjetivamente. Después de todo, lo que realmente importaba era demostrar que el sujeto era el señor del mundo.Y sobre ese principio se construyeron cañones, se hicieron viajes, se ordenaron las finanzas públicas, se reorganizó el Estado, se creó el vapor y las industrias (Picht 1989, Standecker 1991).

Desde la perspectiva de la inicial promesa ilustrada, la sociedad moderna realmente existente fue más hija biológica de Newton que de Descartes, aunque sea este último el que aparece en la partida de nacimiento. Como señaló Ernst Gellner (2005), la modernidad se instaló de hecho y no de derecho, pues no logró justificar sus fundamentos allí donde había querido hacerlo. Esta crisis de la Ilustración estableció y dejó abierta la dualidad entre el fundamento subjetivo o gnoseológico del mundo y el fundamento práctico o técnico de la naturaleza y el orden social. Esa dualidad entre sujeto y naturaleza, conocimiento y poder, sentido y orden, vivida como problema desde la perspectiva de la promesa ilustrada, está en el origen de la modernidad real y la atraviesa hasta hoy como una de sus paradojas más profundas.

Muy pronto se entendió que lo que estaba en juego no era un debate entre escuelas de pensamiento, sino una dualidad que era constitutiva de la nueva realidad que comenzaba a emerger. Por eso se crearon los conceptos que la hicieran observable. Cultura fue uno de ellos, aquel que permitió reconocer la tensión entre subjetividad y orden en el plano de la vida social. Kant fue uno de los primeros en hacerlo:

"Nosotros estamos civilizados hasta el hastío, en todo tipo de actividad y comportamiento social. Pero para considerarnos moralizados, para eso nos falta todavía muchísimo. Pues la idea de la moralidad pertenece aún a la cultura. El uso corriente de esa idea, sin embargo, la cual se refiere sólo a la apariencia del comportamiento correcto en el orgullo y en la corrección exterior, sólo significa simplemente civilización" (Kant 1968 [1784]: 31).

Esta notable cita contiene ya todos los elementos que permitieron al concepto de cultura iniciar su carrera en las Ciencias Sociales: moralidad versus comportamiento, interioridad subjetiva versus exterioridad de las reglas y costumbres sociales, cultura versus civilización. Pero no se trata de una casualidad debida a la genialidad kantiana. Expresa una operación conceptual del período. Ya Samuel Puffendorf en 1686 y Johann Adelung en 1793 - pensadores ilustrados a los que se les suele atribuir el origen del concepto moderno de cultura - habían organizado bajo ese concepto la tensión en la vida social entre naturaleza y moral que había dejado tras de sí la resaca de la razón ilustrada (Böllenbeck 1994, Elias 1989). 
Por supuesto que ni el vocablo, ni algunas de sus distinciones básicas lo inventaron ellos. El concepto de cultura tenía en esa época la no despreciable antigüedad de mil ochocientos años. Permítanme un pequeño rodeo. ¿Qué hizo posible que ese concepto pudiera ser usado para estos nuevos fines? Es una pregunta que no tiene un interés arqueológico, sino teórico. Pues ocurre que los que se apropian de conceptos viejos para decir cosas nuevas suelen quedar teñidos indeleblemente con los colores originales.

Hasta dónde se sabe, el primer uso del término cultura - un termino bastante vulgar referido a algo así como 'pasar el arado' - para referirse a la vida de los hombres aparece en Cicerón en torno al año 100 a.c. Con él se quiere indicar que los hombres no nacen listos para vivir de acuerdo al ideal de la humanidad. Para ello, y como Cicerón era estoico, proponía que cada uno tenía que 'pasarse el arado a sí mismo' para germinar la semilla sublime que portaba en sí. La metáfora agrícola oponía naturaleza biológica frente a los ideales, y el tránsito entre ambos se lo atribuía a la capacidad del hombre para superar su propia naturalidad (Fisch 1992, Williams 1985, Bauman 2002, Dalfen 1984).

Como Cicerón quería proponer una metáfora moral, por supuesto que no usó el término cultura para describir la dificultad y el drama real de semejante tránsito entre naturaleza y humanidad, sino para señalar la obvia superioridad del punto de llegada y la garantía de la viabilidad del paso. ¿Acaso no es normal que una semilla bien 'cultivada' derive en flor? El estoico latino no sólo estableció una oposición, sino que la hizo moralmente asimétrica y transitable con fuerzas humanas. Cultura quedó marcada con la seña de la posibilidad práctica del absoluto moral a partir de la sola fuerza de la interioridad humana.

La historia posterior se encargó de reforzar esa marca. Los monjes medievales tomaron el concepto en su sentido dual y moral pero lo aplicaron exclusivamente al 'culto' ritual religioso. Es decir, las cosas son como las describió Cicerón, pero la fuerza de la interioridad se realiza a través de las ceremonias de la iglesia. Con ello cultura pasó a ser una descripción de lo que ocurría en los conventos y un fundamento de su humanidad superior. Cultura es el reino de los 'spirituali'.

El renacimiento descubrió luego que la vida en la ciudad tenía su propia bondad y legitimidad y que no requería del predominio de los monasterios para desplegarse, sino más bien oponerse a ellos. Para significar ese giro se desempolvó también un antiguo vocablo: 'civilización', el modo de vida de las ciudades. La 'vita civilis' fue opuesta en ese uso al aislamiento de la 'vita spiritualis' y su culto, pues aquella se funda en una razón natural inherente al género humano que es inmanente, a nombre de la cual los órdenes sociales pueden encontrar su perfección y autonomía.Ya no se necesita la cultura, basta la civilización para ser felices (Fisch 1992). 
Al poco andar, sin embargo, a algunos les pareció que las maneras cortesanas que definían el ideal de la vida de las ciudades se habían vuelto algo tan vacío y exterior, tan excluyente en su clausura aristocrática, que no parecía posible que ellas sirvieran para canalizar el tránsito de los hombres hacia su perfección. Pero en un mundo poblado ya por un individuo consciente del valor de su autonomía tampoco era recomendable un retorno a la soledad espiritual de los monasterios. Una de las respuestas fue el 'amour courtois, el movimiento literario de los caballeros marginales. Ellos situaron la contradicción en el centro mismo de la 'civilización': las relaciones eróticas en el marco de la costumbre de los matrimonios arreglados por la corte. Allí van afirmar al amor como un motivo natural absoluto que no puede ser ni dominado ni realizado por la razón natural (en esa época, el orden de las costumbres de la ciudad). En la ciudad el amor sólo puede afirmarse como imposibilidad y tragedia, como distancia de la amada y como conciencia de esa distancia; es decir, como ascética y autocontrol. Sólo la muerte, no las reglas de la ciudad, puede producir la identidad de los amantes, como les hizo saber Shakespeare a sus contemporáneos a través de Romeo y Julieta (Steger 1991).

El romance cortesano, hasta donde sé, no usó el concepto de cultura, pero definió un tipo de subjetividad solipsista, ascética y trágica que radicalizó al extremo la oposición que aquel transportaba y la situó al interior mismo de la sociedad. Ella tendrá una importante influencia en la definición de la idea de subjetividad que formará parte central del futuro concepto de individuo en la modernidad (Martuccelli 2007). También influirá en la formación de alguna de las variantes del concepto de cultura en las Ciencias Sociales.

Un último giro que resulta importante para la historia posterior es el realizado por el historicismo y el romanticismo sobre la oposición entre civilización y cultura elaborada por los ilustrados alemanes. No pasó mucho tiempo antes de que se viera que la fundación de la autonomía del sujeto por la vía de la reflexividad individual conducía a contradicciones cada vez más flagrantes. Tampoco pasó demasiado tiempo antes de que la oposición entre civilización y cultura se transformara en un hecho militar y político por causa, primero, de las guerras entre Napoleón y los Estados independientes alemanes y, luego, por causa de la oposición entre burguesía intelectual y nobleza agraria al interior de éstos (Elias 1989, Bollenbeck 1994).

Aquellos impulsos coincidieron en una reformulación de la teoría ilustrada del sujeto, agregándole la idea de que el fundamento de su autonomía se ha formado históricamente y se expresa en los aspectos lingüísticos y simbólicos de su comunidad de pertenencia. La cultura de la comunidad es el principio de trascendencia en que se funda la subjetividad individual. Este fue el núcleo de la reacción romántica a la crisis de la Ilustración (Safranski 2007). Esta idea, formulada inicialmente por Herder, ha tenido una exitosa carrera en las teorías de la cultura, especialmente a través de dos 
de sus derivados. El primero es la idea de que los pueblos tienen identidad, algo así como la idea clara y distinta de Descartes pero situada ahora en un sujeto colectivo e histórico (Güell 1996). El segundo supuesto se apoya en el anterior y resulta en una afirmación del valor intrínseco e incomparable de cada comunidad cultural histórica, con lo cual no puede ser reducida a un parámetro universalista. En esta afirmación hunde sus raíces el relativismo cultural, que después tendrá una muy exitosa carrera en las ciencias de la cultura (Bauman 2002).

La anterior historia del concepto de cultura - de Cicerón al romanticismo - explica bien las razones que tuvieron para adoptarlo aquellos pensadores que, como Kant, intentaron asegurar la primacía de la subjetividad en el contexto de la crisis del racionalismo y de la creciente autonomía de la razón práctica. Por cierto que no fue el concepto más importante para muchos de ellos, pero les permitió organizar su descripción de la oposición entre sus teorías del conocimiento y la amenazante falta de fundamento y exceso de poder que atribuían a la civilización técnica en gestación.Y el uso de ese antiguo concepto cobró el precio de su marca histórica: entre el sujeto autofundado y la naturalidad de la vida social sólo podía observarse un abismo cada vez más dificil de franquear sin tener que recurrir a los extremos del exilio ascético o de la afirmación de la negatividad radical de la cultura respecto del orden social, como ocurría con la afirmación de la muerte en el amor romántico. Sobre esa historia quedaron puestos los fundamentos para los desarrollos posteriores del concepto de cultura en las Ciencias Sociales. Pero esa historia siguió dos derroteros distintos y se puede, por tanto, reconstruir de dos maneras.

La primera reconstrucción posible consiste en seguir a aquellos que usaron el concepto de cultura para identificar los modos de articulación entre subjetividad y orden social en aquellas sociedades donde no existía el modo moderno de esa escisión. La cultura fue definida como un hecho natural básico de toda organización social y cuyos diferentes modos de darse servían para explicar las diferencias entre las sociedades reales. Ese mismo concepto de cultura fue usado también como parámetro comparativo desde la perspectiva del progreso. Como si desde el punto de vista de la cultura existiera una continuidad, ya sea en un sentido ascendente y progresivo - lo cual permitía explicar la superioridad de la sociedad moderna por la mayor cantidad de componentes racionales en su cultura - o en un sentido de repetición de lo mismo - lo cual permitía señalar la persistencia del fundamento irracional en el seno mismo de la sociedad moderna. En cualquier caso, en esas versiones la articulación entre subjetividad y orden era un hecho asegurado por las dinámicas de la naturaleza o de la historia, de la cual la propia subjetividad, entendida más bien como psicología, era parte intrínseca. Este es, me parece, el camino que en general tomó la antropología (Harris 2005, Luhmann 1995). 
Muy distinto fue el camino seguido por la sociología. En ella la escisión entre subjetividad y orden social fue definida como un hecho histórico específico que caracterizaba a la modernidad. Además, por la forma en que había surgido, era reconocido como un problema de muy difícil solución. La sociología partió definiendo a la cultura como el nombre de un problema, mientras la antropología vio en ella el nombre de una solución. Se trate de Weber, Parsons, Goffman o Luhmann el concepto de cultura servirá para denunciar, mediar, superar o negar la escisión entre la idea de subjetividad y la idea de racionalidad natural del orden social que quedó como herencia de la crisis de la promesa ilustrada.

Cualquiera sea la posición que se tome frente a ella, esa escisión es estructurante del concepto sociológico de cultura y éste siempre está ahí para señalar esa escisión como un problema que debe ser abordado si se quiere explicar cómo es posible la vida social moderna, es decir, cómo es posible que las subjetividades ilustradas vivan en una naturaleza que es autónoma en su racionalidad. El concepto sociológico de cultura, de manera explícita en algunos e implícita en otros, es una perspectiva para formular y enfrentar un problema que permanece una y otra vez sin solución. Cultura en sociología puede ser entendida como el nombre de la modernidad en tanto drama.

\section{La cultura como mediación entre subjetividad y sociedad a partir del sujeto}

A diferencia de los filósofos, los sociólogos fueron realistas respecto de la vida moderna. Sabían que la escisión entre subjetividad y orden social no era un asunto de opciones, respecto de la cual se podía elegir. Su punto de partida era que la escisión era un hecho de la realidad y había que explicarla. Es inevitable que para ello le otorgaran más importancia a un polo que al otro, lo cual depende de qué es aquello qué sienten más amenazado por la existencia misma de la escisión. Unos acentuarán el polo del sujeto, otros el polo del orden, otros el vínculo puramente pragmático entre ambos. Como han señalado algunos autores, detrás de los énfasis específicos que adquieren las teorías puede reconocerse el modo específico de plantear los problemas de las respectivas tradiciones nacionales (Lepenies 1994, Reinwald 1991, Standecker 1991).

En la tradición alemana, tanto por razones de herencia filosófica como de contexto político, lo que importaba resaltar era al sujeto como fuente autónoma y superior de sentido. Georg Simmel y Max Weber son las piedras angulares de esta vertiente del concepto de cultura. En esa tradición, aunque con algunas fuentes exógenas, se desarrollará también la potente teoría cultural crítica de la Escuela de Frankfurt. 
Georg Simmel (1858-1918) formuló una teoría general de las relaciones entre subjetividad y orden social afirmando el carácter universal de la problemática tensión entre ambas. Basado en esa teoría, caracterizó dicha relación en la sociedad moderna como una tragedia. La subjetividad individual, dijo siguiendo a Baudelaire, es un flujo incesante. Por ello, se requiere de formas externas que hagan posible unas relaciones estables con el mundo y consigo mismo. Esto lo demandan las relaciones sociales por la necesidad de objetivaciones que le permitan al individuo constituirse como sujeto y lo demanda la necesidad de un referente estable que aquiete el flujo interior. Simmel no es conservador, sino muy moderno: no hay sujeto sino en las objetivaciones del mundo.

Las formas son objetivaciones de las relaciones sociales reales y éstas siempre están sometidas a la diferenciación de ámbitos del mundo real. Como han de valer para la interacción de muchos a la vez, ellas definen posiciones parciales y abstractas. Frente a ellas, el individuo queda en una relación tensa consigo mismo: entre su demanda intrínseca por reconocerse como totalidad subjetiva y sus fragmentaciones objetivas externas.

En todo proceso de existencia social del individuo se produce la oposición entre el contenido subjetivo y la forma exterior. El primero tiende a su absolutización como principio de sentido y de universalidad, el segundo a su autonomización y a la clausura sobre sí de sus diferenciaciones. Desde la perspectiva del cultivo existencial del sujeto - que es lo que a Simmel le importa -, el paso del individuo a través de la objetivación le permite no sólo constituirse como sujeto. Por su capacidad intrínseca para la reunificación y para dar sentido de totalidad a la exterioridad, el individuo retorna a sí mismo como sujeto efectivamente realizado. Esa dialéctica de mediación entre individuo y forma es la cultura.

"Cultura es el camino desde la unidad cerrada a través de la multiplicidad cerrada, hasta la unidad desarrollada” (Simmel 2002 [1911]:320).

Cultura no es un momento de la forma, un aglutinante simbólico de ella. Para eso Simmel refiere al lenguaje, al dinero o a la religión. Cultura es mediación y, como tal, un campo distinto a lo puramente social.

"Aquí acontece un tornarse objetivo del sujeto y un tornarse subjetivo de algo objetivo, acontecimiento que constituye lo específico del proceso cultural, y en el que por encima de sus contenidos particulares, se muestra su forma metafisica." (Simmel 2002 [1911]:325)

Simmel no desarrolló detenidamente una teoría del funcionamiento de la síntesis cultural, sólo indicó que ella se fundaba en la experiencia de lo universal en lo particular que proporcionaba la razón estética (Walter Benjamín y Theodor Adorno se nutrirán aquí en su reflexión de la modernidad a partir de la cultura o estética de 
masas). Lo interesante es que Simmel pudo atreverse al reconocimiento del carácter compulsivo y alienado de la sociedad porque apostó a la cultura como modo de recuperación del sujeto y como punto de vista moral. Es una apuesta inicial que desembocará en su conocida constatación trágica.

Pero a su juicio, la sociedad moderna se caracteriza por la radical diferenciación y autonomización de las formas. Ello ocurre por su propia tendencia inmanente, por el aumento de la división del trabajo, por la lógica autorreferida de economía industrial y por la extensión y abstracción de la coordinación monetaria. Esto lleva a un mundo objetivo, a una sociedad que es tan diversa en la particularidad de sus objetivaciones que el espíritu subjetivo del individuo no puede ya totalizarlas mediante el trabajo cultural. Por si fuera poco, la vida moderna organiza simbólicamente tal diversidad mediante la autoformación de un pseudo-sujeto totalizador que no es el sujeto espiritual.Aquí Simmel remite explícitamente a la noción de fetichismo de la mercancía de Marx.

De esta manera, y apartándose del punto de vista conservador, afirma que la vida moderna no es ausencia de sentido para la interacción y para la vida objetiva, sino ausencia de sujeto y pérdida del sí mismo. Esta es la tragedia de la cultura en la modernidad, tragedia que se puede formular precisamente gracias al concepto de cultura.

"El hombre se convierte ahora en mero portador de la coerción con la que esta lógica domina los desarrollos y los continúa como en la tangente de la vía por la que regresarían de nuevo al desarrollo cultural del hombre viviente. Esta es la auténtica tragedia de la cultura (...) De ahí surge la situación típica del hombre moderno: estar sumido en un mundo que no carece de significados para él, pero que en el fondo no son plenamente significativos" (Simmel 2002 [1911]:354)

Max Weber (1864-1920) conoció bien las elaboraciones de Simmel, pero buscó y pensó la posibilidad de la autonomía del sujeto moderno por otras vías. Aunque, al final, éste arribará a similar conclusión trágica que aquel. Weber padeció toda su vida bajo el síntoma que Simmel le atribuyó a los individuos de sus teorías: la ansiedad de no poder ordenar racionalmente el mundo y el temor a sucumbir ante el predominio de la irracionalidad. Esto se transformó en el punto de partida de su búsqueda intelectual (Green 1980). En un sentido heroico, siguiendo a su modelo nietzscheano, se propuso la tarea imposible de dotar de sentido al mundo, sin caer ni en el psicologismo que le atribuía a Simmel ni en los particularismos románticos de su época. Tendría que existir una única razón capaz de explicar el sentido del mundo. Aunque el resultado de esta búsqueda fuera trágico desde el punto de vista del individuo, la perspectiva empleada le permitió organizar una síntesis teórica de proporciones canónicas en la cual vinculó historia, sentido subjetivo de la acción y estructura social de una manera probablemente aún no superada. 
En ninguno de sus trabajos Weber desarrolla sistemáticamente el concepto de cultura, aunque éste salpica su obra de principio a fin. En sus escritos se refiere más bien al adjetivo 'cultural' que a 'cultura' en tanto objeto delimitable. La realidad, la que a él le interesa comprender, es en su cuerda más profunda un hecho 'cultural'. De ahí que el análisis del concepto de lo 'cultural' en Weber deba situarse en un nivel distinto al que define el conjunto de las categorías y taxonomías por las cuales se ha hecho conocido. Lo cultural se sitúa en el nivel de su antropología filosófica y requiere ser reconstruido.

Se puede partir por varios lados. En este caso resulta pertinente hacerlo por "La objetividad en las Ciencias Sociales y en la política social" de 1904. En este trabajo Weber se propone encontrar un punto de articulación entre el sentido subjetivo dado siempre en la particularidad histórica y la comprensión de ese sentido por referencia a un punto de vista universal. Esto es lo que él andaba buscando y se propone definir un método para ello. Como no era fácil lograrlo, teniendo en cuenta que ese objetivo estaba siendo cuestionado desde varios frentes, en este trabajo se resumen sus disputas con el historicismo, el psicologismo, el naturalismo positivista y el materialismo histórico. De allí que no es casual la centralidad que adquiere en esta obra lo cultural como punto de partida (Sukale 2006).

La realidad está marcada por la escasez que impide la realización plena de los intereses y necesidades de cada uno. Hay por lo tanto una necesidad de construir 'relaciones sociales' que permitan restringir recíprocamente las libertades y coordinar los intereses. Pero la realidad misma es una masa informe, de una diversidad infinita que cambia permanentemente. Ella no posee en sí misma el orden que haría posible esas relaciones sociales, ni tampoco avanza por fuerza de la historia hacia él. La respuesta a la pregunta cómo se satisfacen las necesidades remite, por tanto, a la construcción de 'relaciones sociales' en una realidad sin orden.

“(...)este caos sólo puede ser ordenado por la circunstancia de que en todo caso únicamente una parte de la realidad particular posee importancia para nosotros, puesto que solo esa parte se halla en relación con las ideas de valor cultural con las cuales abordamos la realidad" (Weber 1974 [1904]: 45).

Esa operación que permite organizar lo real como espacio y como recurso predecible para la acción, y, por lo mismo, como condición de la satisfacción de las necesidades, se llama 'cultura'.

"La cultura es un fragmento finito de entre la incomprensible inmensidad del devenir del mundo, al cual se ha conferido - desde el punto de vista del hombre - un sentido y un significado" (Weber 1974 [1904]: 48).

La realidad cultural, aquella en la cual vive el ser humano, se construye por una atribución de valor. No se trata del valor como referencia de lo bueno, sino como 
ratio, como patrón de medida. La ratio cultural es una organización particular del mundo externo e interno según una atribución causal entre los fenómenos considerados como medios y fines en sentido práctico. Esto remite al liberalismo vitalista de Weber. Los individuos tienen la capacidad y la voluntad para tomar una actitud consciente frente al mundo y conferirle un significado y aquel está, en principio, disponible. La causalidad no es entonces la marca de la servidumbre de la subjetividad frente al mundo, sino el efecto de su libertad, pues ella es la que la construye (Cohn 1998).

El individuo vive en un mundo de diversas organizaciones posibles de los fines y los medios, siendo tarea y posibilidad existencial el conferirles un orden de sentido. De esta manera, cada uno vive en una pluralidad de ratios posibles y al actuar en sociedad es 'portador' de ellas. Para Weber no es existencialmente necesario reducirlas a una unidad, porque la autonomía del individuo - a diferencia de Simmel - no se realiza en el trabajo de totalización, sino en la libertad de significación. Su método de análisis - la comprensión a través de tipos ideales - reproduce el modo cultural de funcionamiento de lo real en varios sentidos. Primero, porque reconstruye las atribuciones de valor y de la ratio derivada de ellas como interpretación del sentido de la acción. Segundo, porque el propio investigador es un sujeto cultural que recorta la multitud de ratios presentes en cada constelación de acción según su propio interés cognoscitivo. Así pues, tanto en su antropología filosófica como en su metodología Weber logró definir a la libertad del sujeto como fundamento de lo real, sin necesidad de recurrir para ello ni a las fuerzas psicológicas ni renegar de la racionalidad intrínseca y objetiva del mundo social. De esta manera, la autonomía del sujeto tiene como condición a la cultura, y ésta al hecho de que el mundo se mantenga abierto a las atribuciones de significado.

¿Donde radica entonces lo específico de la sociedad moderna? Ésta se ha impuesto crecientemente un solo tipo de racionalidad, limitando severamente las capacidades de atribución de sentido de los individuos y por ende su libertad.

"Qué encadenamiento de circunstancias ha conducido a que precisamente sobre el suelo de occidente, y sólo aquí, aparecieran fenómenos culturales, los cuales están en una dirección de desarrollo de significado y validez universal" (Weber 1988a [1920]:1).

La respuesta no debería buscarse en una lógica inmanente del mundo, porque no hay tal cosa. Lo que hay son procesos históricos de atribución de sentido, de imputación de racionalidad. Luego hay que reconstruir aquellos procesos que condujeron a un tipo particular de racionalidad que se hace inmune a su propia historicidad. Esta es la columna vertebral del trabajo de Weber: la reconstrucción del proceso occidental de racionalización como una dinámica cultural específica que conduce a la limitación de las posibilidades culturales. La sociología histórica de Weber está construida para 
interpretar un hecho cultural específico, y éste para dar cuenta de un drama de la cultura en sentido antropológico general.

La unificación del mundo a partir del monoteísmo, la codificación jurídica y musical, la administración del tiempo, la emergencia de una ética intramundana, la significación religiosa del éxito económico, todas ellas creaciones culturales históricas particulares de Occidente que pueden reducirse a tipos ideales, condujeron al creciente predominio de la racionalidad instrumental sobre las otras posibles. Ello explica sólo una parte del destino ambivalente en el que desembocó. La otra parte es ¿por qué esa racionalidad se ha autonomizado al punto que más que una elección posible se impone como única y no deja al individuo otra posibilidad que identificarse con ella?

Esto ha ocurrido porque en Occidente el tipo puro de acción racional con arreglo a fines - que es el modelo del cual se derivan los demás tipos ideales de la acción - no deja espacio a la atribución de sentido. Una vez que se ha puesto el fin en la maximización del logro y al cálculo monetario como ratio, los medios y las reglas de acción pueden ser derivados inequívocamente por la propia lógica de los hechos sin pasar por el trabajo cultural de atribución por parte del sujeto. Con ello la racionalidad instrumental se autonomizó de la cultura, aunque surgió de ella. Lo que partió como algo marginal - la búsqueda de éxito como señal de salvación - se transformó en un fin por sí mismo. La racionalidad, que no puede existir sino como cultura, se transforma en irracionalidad en el momento en que no le deja espacio al trabajo cultural. En esto consiste para Weber la irracionalidad y la tragedia del mundo moderno. No está de más citar otra vez la conocida frase de la conclusión de la Etica Protestante:

“(...) el espíritu se esfumó; el cofre permanece vacío, sin que pueda saberse si para siempre. Como quiera que sea, el capitalismo triunfante, siendo que se apoya en bases mecánicas, ya no requiere más de la ayuda religiosa. Asimismo, es de suponer que se ha extinguido para siempre la rosácea mentalidad de la sucesora del puritanismo, la “Ilustración””. (Weber 1988b [1904/05]:112).

En la obra de Weber, el concepto de cultura se transforma así en el principio heurístico que permite poner en perspectiva antropológica - esto es, de la libertad afirmada como capacidad de atribución de sentido - el significado del proceso de racionalización de Occidente. Desde esta mirada, la razón se descubre al final como irracionalidad.

\section{La cultura como el problema de la mediación entre subjeti- vidad y sociedad a partir de la sociedad}

Hasta aquí no parecieran existir mayores problemas en aceptar la tesis de que en la tradición del idealismo alemán la palabra cultura y el análisis cultural han sido sobre 
todo el nombre de una perspectiva que permite reflexionar sobre la modernidad como escisión problemática entre la autonomía de la subjetividad y el carácter naturalizado del orden social. Es decir, no como un objeto al interior de ese orden, sino como un principio para observar un problema del cual ese orden es uno de sus componentes.

Pero es obvio que la tradición que se desprende del idealismo, o tradición 'subjetiva', hace solo a la mitad del campo del desarrollo, aplicación y significación del concepto de cultura. Existe también otra importante tradición, que podemos llamar 'societaria'. En ella el problema cultural de la modernidad se definirá desde la otra cara de la promesa de la Ilustración; esto es, desde el fundamento autónomo de la integración y reproducción racional del orden social. Esa tradición abordará el problema cultural por la puerta de la autonomía de los sistemas simbólicos que organizan a la sociedad como orden.

No es posible, en el marco de estas notas, reconstruir detenidamente el trayecto de esta segunda tradición. Intentaremos más bien mostrar la plausibilidad de la hipótesis que hemos formulado sobre ella a partir del análisis de uno de los autores que está en sus raíces, que además parece estar en las antípodas de la tradición centrada en la primacía de la subjetividad y que dio origen a una de las corrientes más fuertes en la definición del concepto de cultura: Emile Durkheim.

Emile Durkheim (1958-1917) prácticamente no usó nunca el concepto de cultura y, ciertamente, jamás en un sentido teórico.Y no lo hizo, no porque no lo conociera, pues lo conocía de sobra. De hecho, pasó una temporada estudiando en Alemania la situación de la filosofia y las Ciencias Sociales, publicando dos ensayos al respecto a su vuelta a Francia (Lukes 1984). No lo hizo porque era francés y estaba interesado en enfrentar los problemas de la sociedad moderna experimentados y definidos a la francesa.Y el problema percibido por la intelectualidad francesa a fines del siglo XIX respecto del avance de la modernidad era precisamente opuesto al vivido en Europa central. Allí tenían delante de los ojos la experiencia del terror después de la revolución francesa, repetida en la comuna de París en 1871, la derrota del Imperio francés a manos del alemán en 1870 y la incapacidad de las alianzas de la elite para dotar de estabilidad a la III República. Esos eventos fueron interpretados masivamente como señal de que la razón ilustrada expresada como voluntad libre y utilitaria podía conducir a la violencia y a la desintegración, que la falta de unidad nacional podía conducir a la derrota y que inexistencia de principios de cooperación podía debilitar al Estado (Ramos 1999).

Se trata de una interpretación opuesta a la alemana en lo que respecta a las amenazas de la modernidad. Allí la fuerza cohesiva y moral del orden no eran un problema, sino precisamente lo contrario, la excesiva fuerza y externalidad del orden que amenazaban con ahogar la libertad creadora del individuo. El concepto de cultura estaba 
demasiado anclado, empírica e ideológicamente, en esta definición del problema como para que Durkheim lo usara en su opuesto.

Contrariamente a lo que suele afirmarse, Durkheim no definió ese problema con el concepto de 'civilización', pues éste lo usa para designar la especificidad y límites de un orden social de hecho; en su uso es un concepto descriptivo. Sobre esto fue explícito en un ensayo escrito en 1913, "Notes sur la notion de civilization" (Lukes 1984). La noción que usó como perspectiva y principio heurístico para designar la modernidad como problema fue el de 'moral', que refiere a las fuerzas que cohesionan individuo y sociedad desde la perspectiva de la necesidad de integración de la sociedad. El espejo durkheimiano del concepto de 'cultura' es 'moral' y ambos funcionan en un sentido muy similar. No es casualidad entonces que autores posteriores que trataron de integrar la perspectiva de Durkheim con la de Weber, como Parsons, hayan fundido la idea de moral en la de cultura.

De esta manera, aunque el sociólogo francés no usó el concepto de cultura, muy pronto su análisis del problema moral fue traducido a aquél, dándole una connotación que al poco andar se constituyó en predominante. Ya hacia 1930 etnólogos franceses como Marcel Griaule y Michel Leiris adoptarán el concepto de cultura incorporando en él la tradición proveniente de Durkhein y Mauss (Cuche 2002). Pero esta fusión, que incorporó dos perspectivas opuestas en un mismo concepto, contribuyó en parte también a crear las confusiones conocidas respecto de su delimitación.

Como buen francés de su época, Durkheim - liberal pero algo desconfiado del individuo y, en ese sentido, republicano - definió el núcleo de su preocupación de la siguiente manera:

"Actualmente, nuestro primer deber es hacernos una moral" (Durkheim 1967 [1893]:346).

Normalmente se identifica esta preocupación con la pregunta científica acerca de qué es lo que mantiene unidas a las sociedades. Pero su preocupación era históricamente más específica. Observaba la tensión creciente entre las demandas de libertad y autonomía por parte de los individuos y la fuerte exigencia de coordinación e integración que planteaba la nueva sociedad industrial. Su objetivo era encontrar el punto en el cual la libertad individual se ponía al servicio de la unidad de la sociedad, para asegurar que aquella no fuera destructiva y que esto, a su vez, fortaleciera la libertad del individuo. Es decir, buscaba enfrentar el drama derivado de las amenazas que surgieron tras la autonomización y oposición de las dos fuerzas de la modernidad.

Suele identificarse el conjunto de la propuesta durkheimiana con la tesis de su primera gran obra, "La división social del trabajo" (1893), donde se plantean los pro- 
blemas de la conservación del orden de la sociedad en el tránsito de la solidaridad mecánica a la orgánica. Allí queda establecida de la manera más fuerte su postura 'societaria', es decir, la afirmación de la autonomía y primacía de la sociedad respecto de los individuos. Sin embargo, el problema 'moral' queda lejos de estar resuelto en esa obra y Durkheim seguirá elaborándolo en las siguientes, conciente de la dificultad de cerrarlo de manera satisfactoria. Pero las preguntas, la perspectiva y el dolor de cabeza que le produjo la dificultad para responderlas quedaron instalados ya ahí. Desde la mirada de la hipótesis de estas notas resultará pertinente seguir las huellas de esas dificultades también en las obras sucesivas.

"En lo que refiere al problema que está en los orígenes de esta obra, se trata de las relaciones de la personalidad individual y la solidaridad social. ¿Cómo resulta que el individuo al hacerse más autónomo depende más estrechamente de la sociedad? ¿Cómo puede ser a la vez más personal y más solidario?” (Durkheim 1967 [1893]: $37)$.

En "La división social del trabajo" se preguntó cómo era posible que la fusión entre consciencia y orden que caracteriza a las sociedades premodernas no diera paso a fenómenos masivos de desintegración cuando se debilitaba bajo el aparecimiento de la división del trabajo y de la individuación. Quería además evitar explícitamente las teorías contractualistas, como la de Spencer, que dejaban la solución en manos de la racionalidad con la cual los individuos perseguían sus intereses. Quería evitar también soluciones psicologistas como las que estaban en boga en Alemania.

La respuesta se apoyó en un organicismo evolucionista que otorgó un estatuto de autonomía a la sociedad como hecho 'sui generis'. Por supuesto que esta anterioridad idealista de la sociedad como 'conciencia colectiva' y su afición positivista por los hechos empíricos particulares nunca cuadró muy bien en sus teorías, lo cual solía superar aludiendo a principios electromagnéticos tales como la generación de la energía a partir del roce entre átomos contiguos. Lo que en las contradicciones del concepto alemán de cultura resolvía el espíritu, aquí lo resuelve la electricidad.

En fin, independiente de cómo justificó su origen, el hecho es que afirmada la existencia del 'hecho social', los procesos de integración quedaron en manos de la evolución, aunque una versión de ella que se distanciaba de la idea de la existencia de un universal histórico (Cuche 2002). La evolución aseguraría la adaptación funcional de las partes que se iban diferenciando, creando así un nuevo todo orgánico. Durkheim proponía que si se miraba bien la división del trabajo - esto es poniendo el acento en la dinámica misma de la división, más que en las partes divididas por ella - se podría observar que la especialización aumentaba al mismo tiempo que la complementariedad y mutua necesidad entre las partes divididas. De esta manera, el riesgo de fragmentación quedaba reducido a las formas anómalas de la división del trabajo. 
Pero más importante aún es que la libertad de los individuos, aquella que parecía ser el fundamento del problema, no representaba una contradicción con la unidad a priori del todo social, pues la individuación misma era efecto de la división del trabajo. De esta manera, teóricamente al menos, libertad individual y unidad orgánica de la división del trabajo se reforzaban mutuamente o 'solidariamente'. Con ello la coerción que ejercía la conciencia colectiva en la sociedad premoderna podía ser reemplazada paulatinamente por la interdependencia que crea la división del trabajo y por las normas que la regulan. Aunque este último aspecto es crucial, cómo ocurre el reemplazo de la conciencia colectiva por las normas que coordinan la división del trabajo, no fue resuelto en esta obra (Lukes 1984).

La división del trabajo es una formulación teórica respecto de la forma ideal de la integración de las sociedades modernas. En esta fase Durkheim es optimista pues cree que los aspectos patológicos que observa en su época no se deben a la lógica misma de la división del trabajo, sino a la insuficiencia de su formulación y difusión normativa. Sin embargo, en su investigación sobre el suicidio (1897) su optimismo se atempera. $Y$ es que pareciera que los individuos no se adaptan fácilmente a las normas que aseguran la complementariedad en el seno de la división del trabajo. Ni la razón individual - que se identificaría con las necesidades de complementariedad exigidas por la división del trabajo - ni la fuerza de las normas parece bastar para asegurar la solidaridad de la conciencia con el orden. Ni la coerción exterior de las normas, ni el cálculo interior de la razón pueden asegurar la cohesión de una sociedad diferenciada. Además, para progresar las sociedades requieren dejar espacio suficiente a la anomia, de manera que los individuos puedan desviarse de la norma e innovar, de lo contrario no puede explicarse el cambio. Durkheim buscará entonces distinguir entre la norma como hecho exterior coercitivo y la pulsión interior que lleva a seguirla. La fuente de la moral ha de estar en esta última.

“[...] el deber es la moral en tanto que ordena y prohíbe; es la moral severa y ruda, la de las prescripciones coercitivas; es la consigna la que hay que obedecer. El bien es la moral en tanto que nos parece algo bueno, como una idea amada a la que aspiramos por un movimiento espontáneo" (Durkheim 1963 [1925]: 79).

Con ello redefine los términos de la oposición entre conciencia individual y orden social. Por un lado, se erige la figura de un individuo al que se le reconoce mayor autonomía, pues él no sigue ciegamente la norma, sino que la elige en función del bien que hay en ella. Por el otro, la sociedad ya no existe solo en la dinámica inmanente de la división del trabajo o en la objetividad de las instituciones, sino en un conjunto referencial de normas cuya función depende de su significado subjetivo (Durkheim 2006 [1897]). Consecuentemente Durkheim deja de referirse a la idea de 'conciencia colectiva' que refleja la idea de fusión que caracteriza su primera etapa y pasa a hablar de 'representaciones colectivas', lo cual le permite reconocer una relación más 
mediada y compleja entre individuo y sociedad y, aceptar de paso, que el problema permanece abierto.

¿Cómo cerrarlo de una buena vez? Durkheim hará un intento final, y fundacional, en "Las formas elementales de la vida religiosa" (1912) por explicar cuál es el mecanismo que permite que las elecciones que hacen los individuos en el uso de su libertad sean 'solidarias' con las necesidades de integración moral y coordinación funcional en las sociedades diferenciadas e individualizadas. La respuesta la buscará en una fuerza interior que sea capaz de producir en lo más íntimo de la conciencia individual una compulsión que lleve espontáneamente a elegir a la sociedad por sobre las pasiones egoístas. Creerá encontrarla en un análisis de la estructura profunda de lo sagrado en las sociedades primitivas. Recurriendo nuevamente a las metáforas electromagnéticas, propondrá que en determinadas situaciones de proximidad intensa entre individuos - paradigmáticamente en el ritual - los individuos experimentan el éxtasis de la existencia colectiva. Se trata de una experiencia extraordinaria que no puede deducirse de ninguna psique en particular, sino exclusivamente de las relaciones objetivas al interior de un grupo. Esa energía es reificada y externalizada en la forma de un ser sagrado, que no es otra cosa que la hipóstasis del propio grupo y de su valor subjetivo. Esa es la estructura fundamental que define lo sagrado, es la sociedad transformada en valor trascendente.

"La fuerza religiosa no es otra cosa que el sentimiento que la colectividad inspira en sus miembros, pero proyectado fuera de las conciencias que lo experimentan y objetivado. Para objetivarse, se fija en un objeto que así se convierte en sagrado" (Durkheim 1982 [1912]: 214).

Ese ser divinizado es internalizado luego por el individuo, pero no de cualquier forma, sino en forma de sistemas simbólicos de clasificación. El objeto sagrado se representa en la forma de sistemas de interdicciones. Éstos organizan la realidad de manera dual separando aquellas cosas que pueden ser manipuladas y aquellas que pertenecen al orden profano o instrumental de la vida diaria. Esto es el 'principio totémico', la capacidad de los referentes religiosos para organizar el mundo en lo profano y lo sagrado. Esta distinción es también una hipóstasis de la sociedad. Lo profano es el ámbito de los intereses individuales, lo sagrado el de los sociales. El amor y el temor que provoca lo sagrado son más fuertes que la atracción de lo profano, de tal manera que en caso de contradicción prima la sociedad. En cualquier caso, la contradicción es ahora interior a la consciencia y no remite a la sociedad.

La autonomía de la sociedad respecto de la consciencia se refuerza con la idea de que los sistemas clasificatorios, aún cuando surgen de la interacción y el individuo podría referirlos a sí mismo como su origen, tienen una dinámica autoreferida que les permite, una vez creados, crearse y recrearse en función de lógicas combinatorias propias. (Lukes 1984). Pero la autonomía del individuo queda asegurada por el hecho 
de que estos sistemas de clasificación que le permiten elegir y actuar forman la base misma de la estructura de su conciencia.

Esta aparente solución al problema de la relación entre conciencia y orden dejó varios asuntos pendientes. Por una parte, Durkheim no pudo señalar cuál era el fundamento de los sistemas de clasificación en las sociedades secularizadas. Con ello, no alcanzó a responder por el problema específicamente moderno que lo había llevado hasta la religión. Por la otra, trasladó la escisión entre individuo y sociedad al seno mismo de la estructura de consciencia individual, con lo cual el principio de la libertad, tan caro a él mismo, quedó muy a maltraer. Su solución volvía a quedar abierta, pues había puesto en jaque aquello que constituía uno de los términos del desafio: la libertad individual. Durkhein no derivó en una conciencia trágica. Enfrentado al dilema de esos días, la Gran Guerra, no había ninguna duda dónde radicaba el fundamento de la moral: en el sentimiento patriótico. Dicho lo cual, recuperó el optimismo y se entregó por entero a las tareas de la defensa de la nación francesa (Lukes 1984).

Muchos autores creen ver recién aquí, precisamente cuando él logra aislar los sistemas simbólicos como objeto natural en una idea naturalizada de sociedad, el surgimiento de una sociología durkheiminana de la cultura (Alexander 1988). Esto es razonable, pues es la teoría de la función social de los órdenes clasificatorios la que una corriente hegemónica de la sociología de la cultura - el estructuralismo - adoptó como su punto de partida. Pero es incorrecto, pues la teoría de la función social de la religión formulada por Durkhein es un momento específico dentro de una trayectoria abierta destinada desde su inicio a resolver una de las contradicciones de la modernidad. No es posible, me parece, aislar las investigaciones posteriores sobre la religión de aquella pregunta que ya estaba inscrita en la División del trabajo.

Desde la perspectiva de la hipótesis del significado de cultura como perspectiva para enmarcar y tratar el problema de la modernidad como resultado de la escisión dejada por la crisis de la Ilustración, es interesante describir el isomorfismo entre la definición y solución del problema en Simmel y Weber, por una parte, y en Durkheim por la otra.

Primero, la propuesta ideal de todos ellos es que la reapropiación de lo enajenado (en Simmel y Weber la racionalidad autónoma de la sociedad, en Durkheim la razón egoísta del individuo) se produce a partir de un principio sintético ideal (la cultura en Simmel y Weber, la moral en Durkheim) que es elaborada y portada por un sujeto trascendental (la universalidad de la experiencia estética en Simmel, la atribución de sentido en Weber, la hipóstasis religiosa de la sociedad en Durkheim). En todos ellos el círculo de su razonamiento no cerró como esperaban; a unos se los impidió la fuerza de los hechos sociales y al otro la fuerza del individuo. Los primeros concluyeron reconociendo la tragedia, el segundo confiando en la historia. 
Segundo, el paralelismo se revela también en la relación que establecen Weber y Durkheim entre religión y razón. Ambos sabían que no podía explicarse el mundo moderno a partir de fundamentos puramente modernos, esto es a partir de la simple racionalidad autofundada como en Descartes, ni tampoco a partir de las solas leyes de la naturaleza. En esto ambos son conscientes de que para entender la paradoja en la que viven, es decir, para dotar de un fundamento racional a la sociedad, tienen que recurrir a la religión, aunque considerándola de manera puramente secular. Esto significa el reconocimiento del naufragio en que los ha dejado el temporal de la Ilustración, pero también la decisión de no volver atrás, como lo hicieron muchos conservadores de su época y lo siguen haciendo muchos actuales.

En ambos autores la religión ocupa el lugar central de sus esfuerzos por dotar a la sociedad de fundamento. Weber lo hará a través de la reconstrucción histórica del fundamento religioso del proceso de racionalización occidental; Durkheim a través de una teoría universalista de la función de la religión. El recurso a la religión les significó a ambos reintroducir la compulsividad de la irracionalidad para fundamentar la posibilidad de existencia de una sociedad racional. Durkheim situó a la irracionalidad de la experiencia eléctrica producida por los rituales a la base de los sistemas de clasificación que organizan la racionalidad del mundo desde el punto de vista del individuo. Weber puso a la irracionalidad desde el punto de vista del individuo como la consecuencia inevitable de la organización racional de la sociedad moderna (Gellner 2005). Este es el límite en el que se expresa la sociedad moderna como paradoja, su autonomía racional supone o produce la irracionalidad del sujeto.Y es aquello que se descubre cuando se la mira bajo el prisma de la idea de cultura o de moral, que luego será llamada también cultura.

\section{Apuntes finales}

Espero haber hecho plausible la hipótesis que organiza este texto. En primer lugar, lo que refiere a la comprensión de la modernidad como el problema epistemológico que queda abierto tras la crisis de la Ilustración. En segundo lugar, que la idea de cultura es la perspectiva y espacio de reflexión que han empleado las Ciencias Sociales para pensar la paradoja de la modernidad como problema real de la vida en la sociedad moderna.

Aún así, queda abierta una pregunta: ¿lo que es plausible en el caso de Simmel,Weber y Durkheim siguió siendolo después y es igualmente válido hoy? Mi hipótesis es afirmativa, aunque por cierto escapa al alcance de este texto el comprobarla. Dediquemos, sin embargo, algunos párrafos a delimitarla. Es este sentido, cabe notar que el concepto de cultura y el de moral rebautizado luego como cultura, ha pervivido en su función de análisis de una paradoja tanto en los herederos de la tradición 'subjetiva' como de la 'societaria'. 
Respecto de la tradición 'subjetiva' cabe mencionar a la Escuela de Frankfurt. Ésta transformó el carácter trágico contenido en el concepto de cultura en una reafirmación de la autonomía de la subjetividad, esta vez como utopía. La cultura, si bien no más como el espacio de la síntesis - o de la identidad en los términos frankfurtianos - aún es pensable como el espacio de la experiencia de la 'negatividad' del presente y con ello como punto de partida de la fundamentación de su opuesto utópico: la autonomía del sujeto. La Ilustración queda en esta corriente teórica como una utopía de la identidad, cuya función es puramente negativa (Adorno y Horkheimer 1970, Jay 1974).

Elementos de esta misma tradición, mezclados con Gramsci y otras influencias, han estado presentes también en la primera fase de los 'estudios culturales' británicos. Estos utilizaron el concepto de cultura para precisar el proceso de 'apropiaciones' y 'reinterpretaciones' mediante el cual las clases sometidas en el capitalismo avanzado podían recuperar identidad y autonomía. Visto así, el orden objetivo de la sociedad capitalista no logra eliminar del todo ciertos aspectos de la autonomía de la subjetividad. La dialéctica de la lucha de las interpretaciones permite hacerle algún lugar a la emancipación moderna (Mattelard y Neveau 2004).

En la tradición 'societaria' se puede identificar al estructuralismo de base lingüística que desciende de Durkheim y Saussure. Ahí el problema que el sociólogo francés dejó pendiente en las "Formas elementales de la vida religiosa" es retomado por LevyStrauss mediante la afirmación de la existencia de un código binario natural que es inmanente a los individuos y que permite dotar de orden al mundo. Aquellos actúan racionalmente porque no pueden hacerlo de otra manera y por eso el mundo es inevitablemente racional y consecuente. Con ello se retoma la postura kantiana, pero sin tener que hacer recurso a la reflexividad del individuo. La ratio pertenece a la base lingüística del pensamiento, no al individuo ni al mundo. Sujeto y mundo son diluidos, sólo el mito que los narra tiene fundamento (Levi-Strauss 1968). Sobre estos fundamentos el estructuralismo derivó, por ejemplo en Lacan o Derrida, en el callejón sin salida del encierro del sujeto o del lenguaje sobre sí mismos. Con ello se desmintió la posibilidad de formular a la modernidad como paradoja y, de paso, se develó a la modernidad misma como ilusión. De manera algo previa y paralela, Talcott Parsons (1902-1979), tal vez uno de los sociólogos más influyentes en definir el concepto de cultura realmente existente, intentó resolver - otra vez más se diría - las paradojas de la modernidad. Para ello buscó sintetizar las distintas perspectivas idealismo alemán, organicismo francés y pragmatismo anglosajón - que tematizaban los énfasis opuestos de la modernidad (Parsons 1968 [1937]). Fiel a sus antecesores, él ubicará a la cultura en el punto de fusión de los opuestos enfatizados por esas perspectivas. Cultura será el marco de referencias simbólicas y valóricas que hacen posible el orden de interacciones que define a la sociedad. 
$\mathrm{Al}$ igual que aquellos autores a los que intenta reducir a síntesis, Parsons oscilará en sus énfasis a la hora de definir el concepto de cultura (Habermas 1982, Schmid 1992). En una primera fase hará una afirmación subjetivista de la voluntad que se orienta por las normas referenciales que constituyen la cultura. Luego de ver los problemas que esto le acarreaba, intentó por el otro lado, otorgándole más autonomía al orden del ámbito cultural. Reconoció las reglas propias de consistencia que ha de poseer este orden si se quiere dar cuenta de la integración y estabilidad de la sociedad. La cultura tiene que tener un orden propio distinto a la acción si aspira a ser su principio ordenador. De todas maneras mantuvo su supuesto de que la cultura es un sistema de referencias para el actor que depende de la capacidad de internalización de éste. Finalmente, en una última vuelta de tuerca, extremará la búsqueda de garantías para la función ordenadora de la cultura y basará ésta en un código generativo en parte biológico y en parte lingüístico que opera en los individuos como disposición. De este modo, Parsons oscilará entre la afirmación del individuo que se orienta por normas y la afirmación del sistema que se realiza de manera latente a través de las disposiciones codificadas de los individuos. Respecto de las paradojas de la modernidad, hay que decirlo, no avanzó mucho más que los autores a los que trató de sintetizar.

Erwin Goffmann, (1922-1982) organizador del importante concepto de cultura afirmado en el interaccionalismo simbólico, reaccionará frente a las consecuencias del giro sistémico de Parsons. Apoyado en el idealismo de la fenomenología de Schütz y en el pragmatismo de Mead, repondrá la distancia entre subjetividad y sociedad a partir del concepto de interpretación. La sociedad es el resultado altamente contingente y situacional de los juegos de interpretación recíproca entre los individuos. Logrará con ello reafirmar el carácter intencional de la conciencia individual respecto de su existencia social y, de esta manera, aumentará el margen de su autonomía. Pero su teoría de los 'frames' (Goffman 1977) tendrá grandes problemas para explicar la estabilidad e integración de la sociedad, es decir, para fundar la autonomía del orden.

Como puede verse, lo que cada autor trata de sintetizar bajo el concepto de cultura son los opuestos escindidos de la modernidad. Además, como suelen no lograrlo, oscilan en sus énfasis entre uno y otro polo.Y las teorías de unos autores tratan de compensar a las otras en el reconocimiento de esos opuestos. Suena plausible, entonces, pensar que el sentido y función atribuido al concepto de cultura a partir de Simmel, Weber y Durkheim se mantiene relativamente intacto, con sus contradicciones incluidas, en sus sucesores. A este respecto, es interesante la reacción de Niklas Luhmann (1927-1998) frente a esta historia - que conoce muy bien - pues reafirma nuestra hipótesis por la vía de su negación. Con radical franqueza reconoce que, vista desde una teoría que rescata la racionalidad inmanente del orden social, la síntesis entre subjetividad y sistema o entre subjetividades no es posible. 
"Dicho con toda dureza: queda excluido que el individuo pueda 'formar parte' de la sociedad. No hay comunicación entre individuo y sociedad (...) La sociedad no puede salir de sí misma con sus operaciones y tomar al individuo (...) Que la sociedad no puede operar fuera de sus propios límites es algo que debería ser fácil de comprender, pero ¿por qué no se acepta? Lo mismo vale a la inversa, es decir para la vida y la conciencia del individuo" (Luhmman 1998: 62).

Dado que la síntesis entre subjetividad y orden social concebido como un sistema no es ni siquiera pensable, el concepto de cultura no tiene para Luhmann ningún sentido. En el fondo, el concepto le resulta inofensivo. Si la sociedad es un sistema que crea y administra distinciones arbitrarias y para ello se sirve de palabras que nombran las distinciones, entonces resulta que cultura es una palabra especial que no designa nada, salvo el acto mismo de designar diferencias. (Luhmann, 1995) Esta postura es interesante por su consecuencia, pero también porque su argumento reafirma que el concepto de cultura sólo tiene sentido en el marco de la reflexión sobre la tensión entre subjetividad y orden desde la perspectiva de la promesa de su identidad. Asunto distinto es que él no crea en esta promesa o que no vea el efecto sobre el orden social de que haya gente que si cree en ella o está constituida en ella y actúe en consecuencia. Pero, a pesar de todas las críticas, el concepto se niega a morir. Tal vez precisamente porque hay quienes reconocen que la paradoja de la modernidad no es un punto de vista arbitrario sino un hecho estructurante de la propia experiencia moderna, han iniciado una reciente reposición del concepto de cultura en el seno de las teorías que intentan reformular esa paradoja como la tensión entre estructura y acción (Archer 1988, Giddens 1984, Reckwitz 2006)

Para finalizar, se hace inevitable preguntarse: si efectivamente el concepto de cultura es una perspectiva para nombrar un problema y para otorgarle sentido a una búsqueda, ¿tiene ese concepto alguna posibilidad de servir para describir un hecho empírico? Si la modernidad como hecho real constituye, también como un hecho real, a los opuestos que definen su dinámica - una subjetividad y un sistema que demandan autonomía - y si además aquello queda definido como un problema, es decir, como imposibilidad o carencia, entonces es un hecho empírico que quienes viven y actúan en la modernidad experimentan una paradoja. Entonces, tal vez es posible pensar que el objeto empírico del concepto de cultura remita a la elaboración de la experiencia subjetiva que resulta de vivir en la paradoja de las sociedades modernas, elaboración que, a su vez, hace posible vivir en ella con sentido. Es posible, eso si, que el resultado sea más la reconstrucción de un drama que aquella pacífica certidumbre de la pertenencia y de la identidad que resulta de los conceptos habituales de cultura. 


\section{Bibliografía}

Adorno, T. y Horkheimer, M. (1970): Dialéctica del Iluminismo. Sur, Buenos Aires.

Alexander, J. (1988): Durkheimian Sociology and Cultural Studies Today. En: Alexander, J. (Ed.). Durkheimian Sociology: Cultural studies. Cambridge University Press, Cambridge y Nueva York.

Alexander, J. (2000): Sociología cultural. México, Anthropos.

Archer, M. (1988): Culture and Agency. The Place of Culture in Social Theory. Cambridge University Press, Cambridge.

Bauman, Z. (2002): La cultura como praxis. Paidós, Barcelona.

Bollenbeck, G. (1994): Bildung und Kultur. Glanz und Elend einer deutschen Deutungsmuster. Insel, Frankfurt a. M.

Cohn, G. (1998): Crítica y resignación. Fundamentos de la sociología de Max Weber. Buenos Aires, Universidad Nacional de Quilmes.

Cuche, D. (2002): La noción de cultura en las ciencias sociales. Nueva Visión, Buenos Aires.

Dalfen, J. (1984): Einige Gedanken zum europäischen Kulturbegriff und seinen Wurzeln. En: Paul, S. (Ed.) Kultur. Dietrich Reimer, Berlin.

Bürger, C. y Bürger, P. (2001): La desaparición del sujeto. La historia de la subjetividad de Montaigne a Blanchot.Akal, Madrid.

Durkheim, E. (1967): De la división del trabajo social. Schapire, Buenos Aires.

Durkheim, E. (1982): Las formas elementales de la vida religiosa. Akal, Madrid.

Durkheim, E. (1963): De l'Education morale, PUF, Paris (citado según la traducción de Ramos, R. (1999): 46).

Durkheim, E: (2006): El suicidio. Miño y Dávila, Buenos Aires.

Elias, N. (1989): Über den Prozess der Zivilization. Erster Band. Suhrkamp, Frankfurt a. M.

Fisch, J. (1992): Begriff Kultur. En: Brunner, O; Konze, W.; Koselleck, R., Geschichtliche Grundbegriffe. Band 7. Klett-Cotta, Stuttgart.

Gellner, E. (2005): Razón y cultura. Síntesis, Madrid. 
Giddens, A. (1984): The Constitution of Society. Outline of the Theory of Structuration, Cambridge.

Goffman, E. (1977): Rahmen-Analyse. Ein Versuch über die Organization von Alltagserfahrungen. Suhrkamp, Frankfurt a. M.

Green, M. (1980): Else und Frieda - die Richthofen-Schwestern. Deutscher Taschenbuch Verl, München.

Güell, P. (1996): Historia cultural del programa de Identidad. En: Persona y Sociedad, $X, 1,9-28$.

Habermas, J. (1982): Theorie des Kommunikativen Handels (Band I). Suhrkamp, Frankfurt a. M.

Jameson, F. (1999): El giro cultural. Manatial, Buenos Aires.

Jay, M. (1974): La imaginación dialéctica. Taurus, Madrid.

Kant, I. (1784): Idee zu einer allgemeinen Geschichte in weltbürgerlicher Absicht, En: Werkausgabe, (1968) Bd. XI, Frankfurt a.M. (citado en Reckwitz 2006, trad. del autor).

Kroeber, A. L. y Cluckhohn, C. (1963): Culture: A critical review of concepts and definitions. Vintage, Nueva York.

Lepenies, W- (1994): Las tres culturas. La sociología entre la literatura y la ciencia. Fondo de Cultura Económica, México.

Levy-Strauss, C. (1968): Antropología estructural. Eudeba, Buenos Aires.

Luhmann, N. (1995): Die Kunst der Gesellschaft, Frankfurt a.M.

Luhmann, N. (1998): Complejidad y modernidad. Trotta, Madrid.

Lukes, Steven, (1984): Emile Durkheim. Su vida y su obra. CIS, Madrid.

Martuccelli, D. (2007): Gramáticas del individuo. Losada, Buenos Aires.

Mattelard, A. y Neveau, E. (2004): Introducción a los Estudios Culturales. Paidós, Barcelona.

Parsons, T. (1968): La estructura de la acción social. Guadarrama, Madrid.

Picht, G. (1989): Der Begriff der Natur und seine Geschichte. Klett Cotta, Stuttgart.

Ramos, Ramón (1999): La sociología de Emile Durkheim. CIS, Madrid. 
Reckwitz, A. (2004): Die Kontingenzperspektive der Kultur. En: Jaeger, F, Rüsen, J. (Eds.), Handbuch der Kulturwissenschaften, Bd. III, Stuttgart.

Reckwitz,A. (2006): Die Transformation der Kulturtheorien. Verlbrück, Weilerwist.

Reinwald, H. (1991): Mythos und Methode. Zum Verhältnis von Wissenschaft, Kultur und Erkenntnis. Fink, München.

Safranski, R. (2007): Romantik. Eine deutsche Affäre. Carl HanserVerlag, München.

Schmid, M. (1992): The Concept of Culture and its Place wihtin a Theory of Social Action: A Critique of Talcott Parson's Theory of Culture. En: Münch, R.; Smelser, N., Theory of Culture. University of California Press, Berkeley.

Simmel, G. (2002): Sobre el concepto y tragedia de la cultura. En: Sobre la aventura. Península, Madrid.

Standecker, C. (1991): Symbolwelt und Technik. EberhardVerlag, München.

Steger, H.- A. (1991): Askese und Amour Courtois, , München, EberhardVerlag.

Sukale, M. (2006): Max Weber (1864-1920) Kultur zwischen Kaos und Entzauberung. En: Hofmann, M., Korta, T y Niekisch, S., Culture Club II. Suhrkamp, Frankfurt a. M.

Taubes, J. (2007): Del culto a la cultura. Elementos para una crítica de la razón histórica. Katz, Buenos Aires.

Weber, M. (1974): La objetividad del conocimiento en las ciencias sociales. Editorial de Bolsillo, Barcelona.

Weber, M. (1988a):Vorbemerkung. En: Gesammelte Aufsätze zur Religionssoziologie I. J.C.B.Mohr, Tübingen.

Weber, M. (1988b):La ética protestante y el espíritu del capitalismo. Premia editora, Buenos Aires.

Williams, R. (1979): Politics and Letters. New Left Books, Londres.

Williams, R. (1985): Keywords. Oxford University Press, Nueva York. 